\title{
A statistical analysis of X-ray variability in pre-main sequence objects of the Taurus molecular cloud ${ }^{\star}$
}

\author{
B. Stelzer ${ }^{1}$, E. Flaccomio ${ }^{1}$, K. Briggs ${ }^{2}$, G. Micela ${ }^{1}$, L. Scelsi ${ }^{3}$, M. Audard ${ }^{4}$, I. Pillitteri ${ }^{1,3}$, and M. Güdel ${ }^{2}$ \\ 1 INAF - Osservatorio Astronomico di Palermo, Piazza del Parlamento 1, 90134 Palermo, Italy \\ e-mail: stelzer@astropa.unipa.it \\ 2 Paul Scherrer Institut, Würenlingen and Villigen, 5232 Villigen PSI, Switzerland \\ 3 Dipartimento di Scienze Fisiche ed Astronomiche, Sezione di Astronomia, Università di Palermo, Piazza del Parlamento 1, \\ 90134 Palermo, Italy \\ ${ }^{4}$ Columbia Astrophysics Laboratory, Mail Code 5247, 550 West 120th Street, New York, NY 10027, USA
}

Received 14 July 2006 / Accepted 28 August 2006

\begin{abstract}
Context. This work is part of a systematic X-ray survey of the Taurus star-forming complex with XMM-Newton.

Aims. We study the time series of all X-ray sources associated with Taurus members, to statistically characterize their X-ray variability, and compare the results to those for pre-main sequence stars in the Orion Nebula Cluster and to expectations arising from a model where all the X-ray emission is the result of a large number of stochastically occurring flares.

Methods. The analysis of the light curves is based on a maximum likelihood algorithm that segments the time series in intervals of constant signal without the need of binning. Flares are defined with criteria that take into account the amplitude and the derivative of the segmented light curves. Variability statistics are evaluated for different classes of pre-main sequence stars (protostars, cTTS, wTTS, brown dwarfs), and for different spectral type ranges. Flare frequency and energy distribution are computed.

Results. We find that roughly half of the detected X-ray sources show variability above our sensitivity limit, and in $\sim 26 \%$ of the cases this variability is recognized as flares. Variability is more frequently detected at hard than at soft energies. The variability statistics of cTTS and wTTS are indistinguishable, suggesting a common (coronal) origin for their X-ray emission. The frequency of large flares $\left(E>10^{35} \mathrm{erg}\right)$ on Taurus members is 1 event per star in $800 \mathrm{ks}$. The typical duration of these flares - probably biased by the finite observing time - is about $10 \mathrm{ks}$. We have applied a rigorous maximum likelihood method in the analysis of the number distribution of flare energies on pre-main sequence stars for the first time. In its differential form this distribution follows a power law with index $\alpha=2.4 \pm 0.5$, in the range typically observed for late-type stars and the Sun.

Conclusions. The signature of the X-ray variability in the pre-main sequence stars in Taurus and Orion provides twofold support for coronal heating by flares: (i) the correlation between the maximum variability amplitude and the minimum emission level indicates that both flare and quiescent emissions are closely related to the coronal heating process; (ii) the power law index $\alpha$ derived for the flare energy distribution is large enough to explain the heating of stellar coronae by nano-flares $(\alpha>2)$, albeit associated with a rather large uncertainty that leaves some doubt as to this conclusion.
\end{abstract}

Key words. stars: activity - stars: coronae - stars: pre-main sequence - stars: late-type - X-rays: stars

\section{Introduction}

$\mathrm{X}$-ray variability is a characteristic property of magnetically active stars, including such diverse objects as the Sun, dMe flare stars, RS CVn variables, and pre-main-sequence (pre-MS) T Tauri stars (Feigelson \& Montmerle 1999; Favata \& Micela 2003; Güdel 2004). Variability on long timescales may comprise modulation by rotating active regions (with timescales on the order of days corresponding to the rotation period of the star) or even activity cycles (with expected timescales on the order of years); see Marino et al. (2003); Favata et al. (2004) for examples. Most of the observed variations have short timescales (on the order of hours), are accompanied by an increase in electron temperature, and are therefore attributed to flares resulting from magnetic reconnection events.

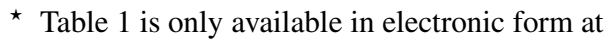
http://www. aanda.org
The intense X-ray radiation from flares is ascribed to the filling of magnetic loops with heated gas that has been driven from the chromospheric layers along the magnetic field lines into the corona (the "chromospheric evaporation scenario", e.g., Antonucci et al. 1984). Various authors have therefore speculated that the overall X-ray emission seen in magnetically active stars is in fact the result of a large number of stochastically occurring flares. In analogy to the "microflare heating" hypothesis in solar physics (see e.g. Hudson 1991), these events are believed to be distributed in energy such that the large majority releases only small amounts of energy, making their individual identification in light curves impossible, while we only measure the integrated emission (e.g., Audard et al. 2000; Güdel et al. 2002, 2003).

Extensive studies yielding statistical properties of X-ray variability for different classes of active stars have long been impeded, mostly due to observational restrictions deriving from 
insufficient monitoring time. Further limitations, besides sensitivity, are imposed by the poor temporal coverage of X-ray instruments in low-Earth orbits that result in frequent occultations of the target by the satellite revolution. XMM-Newton and Chandra have opened the field for systematic investigations on timescales typical for magnetic activity. This is due to the eccentric orbits of both satellites, enabling uninterrupted observations of up to $\sim 2 \mathrm{~d}$. Even then, variability studies are difficult, because in practice it is nearly impossible to maximize both sample size and exposure time per target. An outstanding study in this respect is the nearly 13-d-long observation of the Orion Nebula Cluster (ONC) by Chandra, termed the Chandra Orion Ultradeep Project (COUP). The COUP yielded not only the longest uninterrupted X-ray time series for pre-MS stars so far, but owing to the dense population of the ONC, it also allowed for the examination of the X-ray variability of the largest sample of pre-MS stars in a homogeneous way: More than 1600 $\mathrm{X}$-ray sources, mostly members of the $\mathrm{ONC}$, were detected in a single Chandra field. Amongst others, the COUP resulted in a systematic study of flares on "young Suns" (Wolk et al. 2005), rotational modulation (Flaccomio et al. 2005), the geometry of flare loops (Favata et al. 2005), and X-ray variability of hot stars (Stelzer et al. 2005).

In contrast to the ONC, the Taurus star-forming complex spans a large angle on the sky (see Fig. 1 in Güdel et al. 2007). This implies that observing a significant fraction of its stellar members with a telescope operating in pointing mode requires many different exposures. Realistically, the whole population can be sampled only by an all-sky monitoring. Neuhäuser et al. (1995) have used the data obtained during the ROSAT AllSky Survey (RASS) to study the X-ray emission of the preMS stars in Taurus. However, the RASS consisted of snapshots of a few seconds each separated by $\sim 90 \mathrm{~min}$, corresponding to the Earth revolution of the ROSAT satellite, and is inappropriate for timing studies. A systematic study of the X-ray variability of the pre-MS stars in Taurus-Auriga based on more than 100 pointed ROSAT PSPC observations was presented by Stelzer et al. (2000). That investigation yielded the first estimate of flare rates for these stars, which were found in outburst during about $1 \%$ of the observing time. Various uncertainties compromise this measurement, e.g., the ROSAT data set was (i) inhomogeneous in sensitivity due to the vastly different exposure times of the individual observations; (ii) affected by Earth blocks due to the satellite's revolution; and (iii) limited to soft energies.

Here we present a variability study for the pre-MS stars in the Taurus region based on extensive XMM-Newton observations. We discuss data from the XMM-Newton Extended Survey of the Taurus Molecular Cloud (XEST). This project was devised to comprise observations with roughly uniform and uninterrupted exposures of $\sim 30-40 \mathrm{ks}$. Thus, it represents a major improvement with respect to previous X-ray data of the Taurus Molecular Cloud (TMC). A further advantage is the extension of the hard energy band beyond $2 \mathrm{keV}$. A detailed description of the XEST observations is found in Güdel et al. (2007). Here we present a systematic time series analysis of the pre-MS population detected in the XEST.

In Sect. 2 the methods used in the analysis of the time series are described, and the results are summarized. Section 3 deals with a quantitative investigation of the light curves. The relatively short duration of the XEST observations imply that the most likely cause of the variability is flaring. We introduce our way of defining flares and present the results from the flare detection process in that section. Variability statistics for different classes of pre-MS stars in the TMC are given in Sect. 4, including a discussion of observational biases. The frequency and the energy distributions of the detected flares are examined in Sects. 5 and 6. Section 7 presents a detailed comparison of the variability characteristics of cTTS and wTTS. We summarize our results in Sect. 8.

\section{Time series analysis}

The analysis presented in this paper includes the TMC members of all XMM-Newton fields from the XEST, i.e., the observations from our survey with $X M M$-Newton and the observations added from the XMM-Newton archive (see observing log in Table 1 of Güdel et al. 2007), except field No. 1 (T Tau) and No. 25 (AA Tau), which are dedicated to separate projects (Güdel et al., in prep.; Grosso et al., in prep.). Some fields are spatially overlapping, such that several stars are detected in more than one exposure. In XEST fields No. 23 and No. 24, especially, the satellite was pointing at the same sky position and the two exposures are adjacent in time (separated by only $\sim 1 \mathrm{ks}$ ). Therefore, their data was merged for the time series analysis. The total number of X-ray sources in all studied XEST fields that are identified with known TMC members after merging of XEST-23 and XEST-24 is 126 .

The time series analysis is based on the EPIC/pn photon event lists for the individual X-ray sources. There are three exceptions where we resort to the MOS data: (i) for XEST field No. 26 because there is no EPIC/pn data; (ii) for sources that are outside the field-of-view of EPIC/pn; and (iii) sources located on a chip gap resulting in a major loss of photons in EPIC/pn.

The photon extraction regions for source and background photons have been defined taking care to avoid contributions from adjacent sources. The procedure is described in detail by Güdel et al. (2007). For each X-ray source detected in both XEST-23 and XEST-24, the event lists of the source and of the background of both observations were merged. For a given source, the original photon extraction areas in XEST-23 and in XEST-24 may be slightly different. To avoid introducing artificial variability, for each star we selected the smaller of the two extraction regions for both exposures.

The statistical methods we used are independent of data binning, and are described below. The analysis was carried out in different energy bands: $0.3-7.8 \mathrm{keV}$ (broad), $0.3-1.0 \mathrm{keV}$ (soft), and $1.0-7.8 \mathrm{keV}$ (hard).

\subsection{Maximum likelihood blocks}

We searched for variability in each photon time series using a maximum likelihood algorithm. The technique was described by Wolk et al. (2005), who have used the same method for an X-ray variability study of a subsample of the ONC members detected in the COUP. It is derived from the Bayesian blocks described by Scargle (1998). In brief, the time series is split into periods of constant signal (so-called "segments" or "blocks") under the assumption of Poisson noise, by searching iteratively for change points in the intensity level. In contrast to conventional light curve analysis, this method works directly on the sequence of photon arrival times without the need for binning, such that ambiguities introduced by the choice of bin size and bin start are avoided. The maximum likelihood block (MLB) algorithm has two free parameters, the minimum number of counts per segment $\left(N_{\min }\right)$ and the confidence level $(C L)$ for the established change points. The significance thresholds for the change points were determined for any given $N_{\text {min }}$ through extensive 
simulations of constant light curves (Flaccomio et al., in prep.). The results presented throughout this paper are for $C L=99.9 \%$ and $N_{\min }=20$. Now we proceed to a justification of this choice and a detailed description of the data analysis steps.

While the background of Chandra is very low and can be approximated by a constant, in XMM-Newton observations the background is non-negligible and variable. This complicates the analysis. To take the background and its variability into account we have extended the method with respect to the use for the COUP. In short, the principle of this procedure is to create a background subtracted "source-only" or "net source" events list by removing individual photons from the events list of the source position. Subsequently, the variability tests can be applied to this net source events list.

In practice, for the treatment of the background we proceed in the following way for each source events list and the associated background events list: first, the MLB algorithm is applied to the time series for the background, i.e., the background events list ("B") is divided into ML blocks by identifying change points in the background level. The question is then how to subtract the background from the events detected at the source position (which at this point comprise both source and background photons; ="S+B""). We first scale the segmented background to the extraction area of the source. This gives the expected number of background events at the source position for each segment in which the background is constant. The respective number of photons is then subtracted from the " $\mathrm{S}+\mathrm{B}$ " " events list uniformly within each time-interval of constant background. The result is a background subtracted source-only events list ("S"), ready for further analysis.

The MLB algorithm is now applied to " $\mathrm{S}$ " to yield the segmented source light curve. When computing the errors of the count rate in each segment, the (subtracted) number of background photons within the respective time segment is considered. Note that, obviously, the segments of " $\mathrm{S}$ " are different and independent from the segments of "B".

In general, a source can be considered variable at a certain confidence level if the background-subtracted blocked light curve consists of more than one segment. In the course of the analysis it was noticed that for some sources the detected variability coincides with times of very high and variable background. These variations could be a result of inaccurate background subtraction. To check if the variations in these critical time intervals are spurious, we simulated constant light curves by generating a random distribution of photon arrival times within a time interval corresponding to the length of the observation. The number of photons randomly generated was chosen such that the count rate of the simulated data is equal to the average net count rate of the observed source. Then, we superposed the observed area-scaled background on the simulated source events list, and carried out the background subtraction and MLB analysis. The procedure for the treatment of the background is analogous to the one described above for the observed data, i.e., the background was subtracted blockwise from the simulated " $\mathrm{S}_{\text {sim }}+\mathrm{B}$ " events list and a background subtracted simulated " $\mathrm{S}_{\text {sim }}$ " events list was computed.

For each source, 5000 simulations were performed, with $C L=99.9 \%$ and $N_{\min }=20$. Then the blocked light curves were examined. The simulated data sets are expected to yield no change point in the MLB analysis. However, in a substantial number of sources where the algorithm detected variability in the observed data, variability was also detected in a high fraction $(\gg 10 \%)$ of the simulated light curves. These cases are found among faint sources that have high and strongly variable background, and the statistical subtraction of background photons is problematic.

To avoid such detections of spurious variability, for the ultimate analysis of the data we excluded all time intervals corresponding to blocks from " $\mathrm{B}$ " in which the number of events in the background after scaling to the source area is three or more times higher than the number of events in the source. In general, the reduction of the exposure time by this "high-background filter" is low, less than $10 \%$ for 105 of 126 sources. The worst cases occur in XEST-03 and XEST-24, where the background is so high, that - depending on the source brightness - up to $40 \%$ of the observing time was removed.

Residual variability imposed by the background can be assessed with the help of the simulated data. Clearly, the effect of background induced variations is to decrease the significance of any detected variability with respect to the chosen $C L$. In practice, we use the fraction of simulated event lists that appear non-variable with the MLB method to define a "corrected" confidence level $C L_{\text {corr }}$ for the variability detected in the observed data. We delay the discussion of the results to Sect. 4, and proceed first with the description of the analysis steps.

The analysis was performed in all three energy bands (soft, hard, and broad) with values for $N_{\min }$ of 1 and 20 , henceforth referred to as MLB 1 and MLB 20, respectively. Higher values for $N_{\text {min }}$ can be used, e.g., to define time segments for time resolved spectral analysis (see Franciosini et al. 2007). A low number of the allowed minimum counts per block favors the detection of variability in faint sources, but also the detection of spurious variations, that arise, e.g., from residual background contamination. A light curve with an example for this latter effect is shown in Fig. 1; see figure caption for further explanations.

A careful comparison showed that the variability statistics obtained with MLB 1 and with MLB 20 are widely consistent. For seven sources the segmentation of the broad band is different for MLB 1 and MLB 20. Among these, three sources are constant for $N_{\min }=20$, but one change point is found with MLB 1 . Since these three sources are very faint, the detection of variability is hampered for MLB 20 (because the total number of net source counts is only slightly higher than the change point threshold $N_{\min }=20$ ). However, in practice, their variability detected with MLB 1 is likely spurious because it is related to times of high background not rejected by our high-background filter; see example in Fig. 1. For the other four sources at least one change point is detected with both MLB 1 and MLB 20, and only the number or position of the change points is different between the two tests. To conclude, we find no case where the detection of variability is impeded with MLB 20 due to the higher threshold for the minimum number of counts per segment with respect to MLB 1. Based on these results, we decided to present the results of the analysis with $N_{\min }=20$ in this paper.

\subsection{Kolmogorov-Smirnov test}

As an additional investigation of the variability we performed a Kolmogorov-Smirnov (KS) test on the background subtracted events lists. The output of the KS test is a probability $P_{\mathrm{KS}}$ that the variability detected in the observed data is physical. Since the "S" events file has been manipulated by the subtraction of the background, it is not immediately obvious that the KS test is applicable. To examine the validity of the $\mathrm{KS}$ analysis, we made use of the simulations of the constant source event lists described above. Analogously to the MLB analysis, the simulated light curves should be invariable against the KS test, except for spurious variability detections due to random fluctuations in 
XEST-19-009



XEST-19-009

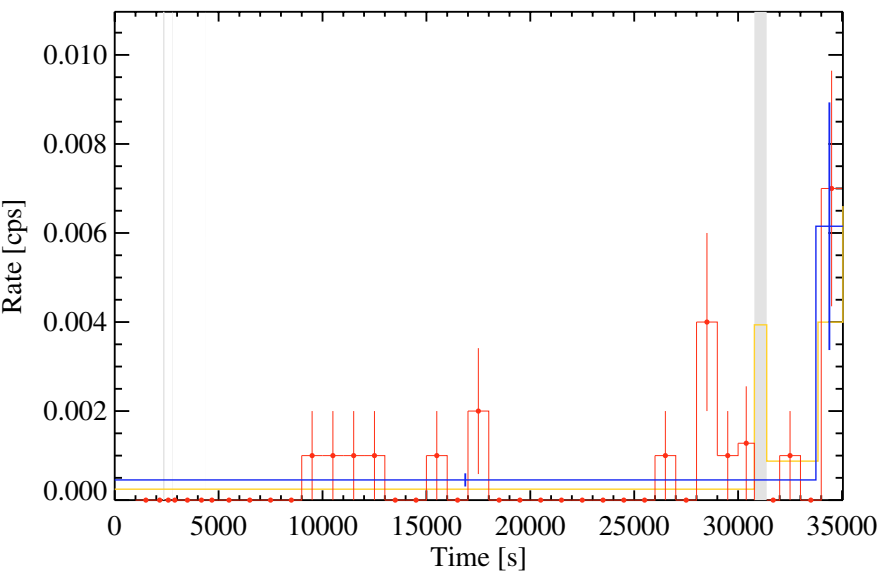

Fig. 1. Broad band EPIC/MOS 1 light curve of XEST-19-009. The background subtracted source signal is shown in two representations: binned into $1000 \mathrm{~s}$ intervals (red) and segmented with the MLB algorithm (blue). The left panel shows the segmentation obtained with $N_{\text {min }}=20$ (only one block, i.e., constant light curve); the right panel shows the segmentation resulting from $N_{\min }=1$ (two blocks, i.e., variable light curve). The background segments scaled to the source area are shown in yellow. Time intervals rejected by our high-background filter are represented by grey-shaded areas. The division into two blocks with MLB 1 probably results from imperfect background subtraction.

the photon arrival times and additional noise introduced by the background subtraction. To quantify the effect of such spurious variations, for each variable source, we computed the fraction $f_{\mathrm{P}}$ of simulated light curves that show a probability $P_{\mathrm{KS}}$ of being variable higher than the value derived for the observed photon time series. The "effective" probability for variability in the data is $P_{\mathrm{KS} \text {,eff }}=1-f_{\mathrm{P}}$.

\subsection{Results from variability tests}

The variability analysis described in the previous section was applied to 126 photon time series from the 122 different TMC members detected in the XEST. The results are given in Table 1 for all examined sources. For a few sources, in the soft and/or hard bands the variability tests could not be carried out due to poor statistics. The entries in Table 1 are given as in Güdel et al. (2007) in order of increasing right ascension. We list the XEST No. (Col. 1), identification (Col. 2), classification defined by Güdel et al. (2007) that combines the Young Stellar Object class derived from the infrared spectral energy distribution and the $\mathrm{T}$ Tauri type based on $\mathrm{H} \alpha$ equivalent widths (Col. 3), and the spectral type (Col. 4). More details concerning the definitions of the stellar parameters are found in Güdel et al. (2007). Column 5 gives the broad band exposure time. Generally, the instrument used is EPIC/pn; the exceptions are indicated by labels "M1" for MOS 1 and "M2" for MOS 2 at the end of Col. 5. The remaining columns represent the results of the variability tests. For all three energy bands the number of source photons ("Cts"), the number of segments resulting from the MLB analysis (" $N_{\mathrm{b}}$ "), the confidence level of the MLB analysis (" $C L_{\text {corr }}$ "), and the probability for variability according to the KS test $\left(P_{\mathrm{KS} \text {,eff }}\right)$ are given; see table caption for more details.

We recall that the parameter describing the variability according to the MLB method $(C L)$ is a threshold, indicating a lower limit for the significance (or confidence) with which the detected variability is physical, i.e., not spurious. The parameter describing the variability according to the KS test $(P)$ is a probability for source variability. Therefore, variability detected with a low value for $C L$ is compatible with a high value of $P$.
In general, we find good agreement between the detection of variability with the KS test and with the MLB method. In this article we distinguish variable from non-variable sources based on the results from the MLB analysis for the broad band. There are 67 sources with a number of blocks $N_{\mathrm{b}}>1$. For most of these sources the KS test probability for variability is very high. Exceptions are XEST-19-049 and XEST-08-058, with $P_{\mathrm{KS}}<0.95$. After visual inspection of their light curves we assigned these two sources to the non-variable sample. Then, the total number of variable TMC sources is 65 , representing $65 / 126=52 \%$ of all TMC members detected in the XEST.

In Fig. 2 we display the light curves of three TMC members, representing some of the typical observed shapes: fast "impulsive" variations, on the timescale of hours carrying the signature of flares (e.g., XEST-14-006), gradual variations possibly representing a fraction of a long-duration event (e.g., XEST-15-040), and constant emission (e.g., XEST-22-021).

\section{Quantifying variability: definition of flares}

To classify the variability we examined the amplitudes and the timescales involved. Wolk et al. (2005) have divided the segments resulting from a similar MLB analysis into three different types, representing "characteristic", "elevated", and "very elevated" intensities. The relatively short exposure time of the XEST observations together with the typical strong variability of young stars makes it difficult to tell what the characteristic emission level of a given source is. Therefore, we follow a somewhat simplified scheme, in which we consider the segment with the lowest count rate in a given time series as the "characteristic" emission. The characteristic count rate is henceforth denoted $R_{\mathrm{ch}}$.

Similarly to Wolk et al. (2005), for the definition of flares we make use of the amplitude and the derivative of the segmented light curve. The amplitude is defined as

$A_{i}=\frac{R_{i}-2 \sigma_{i}}{R_{\mathrm{ch}}+2 \sigma_{\mathrm{ch}}}$ 


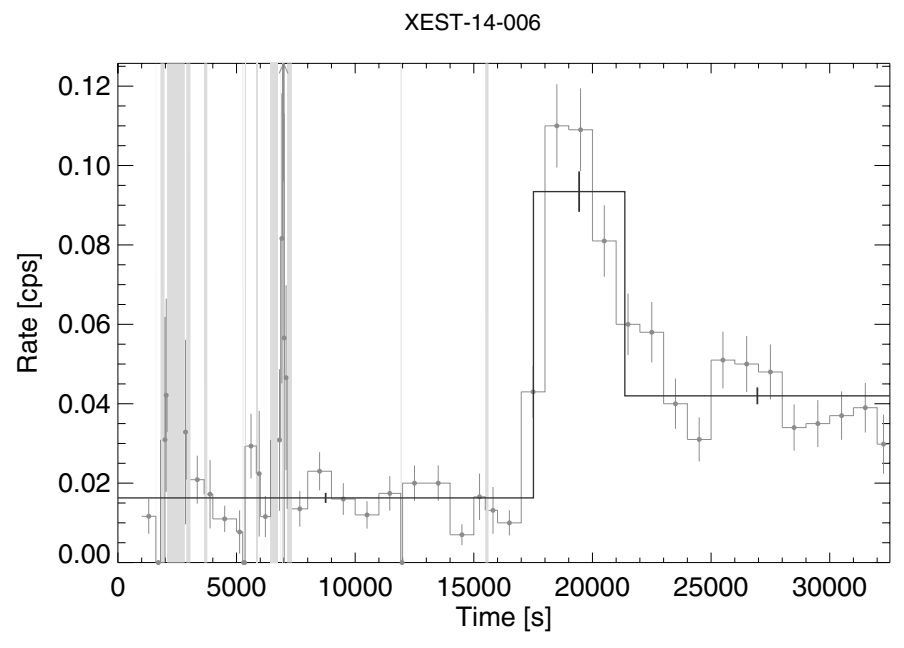

XEST-22-021

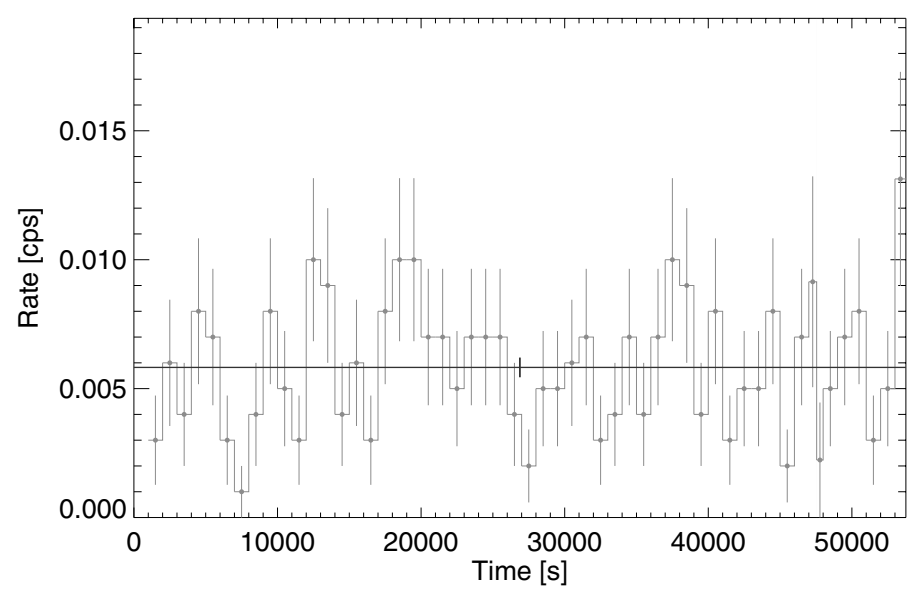

and the derivative as

$\Delta_{i+1}=\frac{\left(R_{i+1}-R_{i}\right) / R_{i}}{\operatorname{MIN}\left[t_{i+1}, t_{i}\right]}\left[\mathrm{s}^{-1}\right]$.

In Eqs. (1) and (2), $R_{i}$ is the count rate of segment $i, \sigma_{i}$ its uncertainty, and $t_{i}$ its duration.

We define those variability detections for which both of the following two criteria are fullfilled as a flare: (i) the amplitude $A_{i}>1.5$ in one or more consecutive segments; and (ii) the maximum of the derivatives in these segments exceeds the threshold of $\Delta_{i}>5 \times 10^{-5}$.

\subsection{Results from flare detection}

With the procedure described above flares are detected on 30 of the 65 variable sources. Two sources have shown more than one event (XEST-23-047 with two flares, and XEST-26-067 with three flares). The results of the flare detection process are summarized in Table 2. For all flaring sources the XEST ID (Col. 1), the amplitude of the flare $A_{\mathrm{F}}$ (Col. 2) and the maximum of the derivative $[\operatorname{MAX}(\Delta)]_{\mathrm{F}}$ during the flare (Col. 3) are given. Furthermore, we list the duration $\tau_{\mathrm{F}}$ (Col. 4), obtained by summing the length of all segments that define the flare. Owing to the short exposure times, only 18 events are observed in their entirety. For the remaining flares the duration given in Table 2 is a lower limit. In Col. 5 the quiescent luminosity $L_{\mathrm{ch}}$ is given, and Cols. 6 and 7 represent the flare luminosity $L_{\mathrm{F}}$ and flare energy $E_{\mathrm{F}}$.



Fig. 2. EPIC/pn time series for three TMC members, demonstrating the various shapes of the light curves: a "canonical" flare (XEST-14-006), a smooth decrease of the count rate (XEST-15-040), and a constant signal (XEST-22-021). The blocks resulting from the MLB analysis are overlaid on the binned light curve (1000 s bins). Data is background subtracted. Time intervals removed by the background filter are marked with grey-shades. For clarity the background light curve is not shown.

Before the conversion to luminosities, the count rates were multiplied with a correction factor that accounts for the source photons outside the extraction area and for the vignetting. Then PIMMS $^{1}$ was used to obtain the unabsorbed flux for a given count rate under the assumption of a 1-T Raymond-Smith model (Raymond \& Smith 1977) subject to photoabsorption. For the column density and temperature of the model we adopted the results from the spectral fitting presented in Table 6 of Güdel et al. (2007) for each individual X-ray source. In the case of 2-T spectral fits we computed the emission measure weighted mean temperature for the input to PIMMS. The flux was converted to luminosity assuming a distance of $140 \mathrm{pc}$ for all sources.

This procedure applied to the characteristic rate $R_{\mathrm{ch}}$ yields $L_{\mathrm{ch}}$, while from the average count of all segments that define the flare we obtain the average source luminosity during the flare. The flare luminosity $L_{\mathrm{F}}$ was obtained by subtracting $L_{\mathrm{ch}}$ from the average source luminosity during the flare. Finally, the flare energy was computed by multiplying $L_{\mathrm{F}}$ by $\tau_{\mathrm{F}}$. In cases where only the rise or only the decay were observed, $E_{\mathrm{F}}$ is a lower limit to the energy emitted during the event.

The last column of Table 2 provides a flag that characterizes the shape of the light curve for the brightest sources with flares, adopted from Franciosini et al. (2007). They present a detailed study of the time-evolution of spectral parameters for the brightest variable XEST sources, and classify them into three

\footnotetext{
1 The Portable Interactive Multi-Mission Simulator (PIMMS) is accessible at http: //asc . harvard.edu/toolkit/pimms . jsp
} 
Table 2. Parameters of X-ray flares detected on TMC members during the XEST: amplitude $A_{\mathrm{F}}$ and derivative $[M A X(\triangle)]_{\mathrm{F}}$ are used to identify flares; the duration $\tau_{\mathrm{F}}$, characteristic luminosity $L_{\mathrm{ch}}$ and flare luminosity $L_{\mathrm{F}}$, and the flare energy $E_{\mathrm{F}}$ are derived as described in Sect. 3.1; the last column provides a flag that classifies the shape of the lightcurve (see Franciosini et al. 2007).

\begin{tabular}{|c|c|c|c|c|c|c|c|}
\hline $\begin{array}{l}\text { XEST } \\
\text { No. }\end{array}$ & $\overline{\overline{A_{\mathrm{F}}}}$ & $\begin{array}{c}{[\operatorname{MAX}(\Delta)]_{\mathrm{F}}} \\
{\left[10^{-5} \mathrm{cts} \mathrm{s}^{-2}\right]}\end{array}$ & $\begin{array}{r}\tau_{\mathrm{F}} \\
{[\mathrm{ks}]}\end{array}$ & $\begin{array}{l}\log L_{\mathrm{ch}} \\
{[\mathrm{erg} / \mathrm{s}]}\end{array}$ & $\begin{array}{l}\log L_{\mathrm{F}} \\
{[\mathrm{erg} / \mathrm{s}]}\end{array}$ & $\begin{array}{r}\log E_{\mathrm{F}} \\
\text { [erg] }\end{array}$ & Class \\
\hline $02-022$ & 3.0 & 21.5 & 9.3 & 29.3 & 29.5 & 33.5 & \\
\hline $03-022$ & 2.5 & 9.1 & 13.5 & 29.8 & 30.0 & 34.1 & \\
\hline 04-016 & 2.8 & 27.0 & $>11.5$ & 30.7 & 30.8 & $>34.8$ & smooth \\
\hline $05-024$ & 4.8 & 90.5 & $>1.5$ & 30.3 & 30.9 & $>34.1$ & \\
\hline $07-041$ & 3.7 & 98.8 & 2.7 & 29.7 & 30.1 & 33.5 & \\
\hline 09-026 & 1.9 & 6.0 & $>4.4$ & 31.0 & 31.0 & $>34.6$ & smooth \\
\hline $11-057$ & 6.3 & 48.2 & $>16.6$ & 30.4 & 30.9 & $>35.1$ & smooth \\
\hline $13-004$ & 3.4 & 45.0 & $>7.2$ & 29.3 & 29.6 & $>33.5$ & \\
\hline $14-006$ & 5.7 & 123.0 & 15.0 & 29.8 & 30.2 & 34.4 & \\
\hline $15-040$ & 2.6 & 5.8 & $>20.7$ & 30.8 & 30.8 & $>35.2$ & smooth \\
\hline $17-058$ & 2.6 & 7.2 & $>5.5$ & 29.6 & 29.7 & $>33.5$ & \\
\hline $17-066$ & 3.0 & 95.5 & 9.5 & 30.3 & 30.6 & 34.6 & atypical \\
\hline $18-004$ & 4.0 & 31.1 & $>6.0$ & 29.7 & 30.2 & $>34.0$ & \\
\hline $20-005$ & 1.8 & 7.1 & $>1.2$ & 30.7 & 30.6 & $>33.7$ & \\
\hline $20-022$ & 80.3 & 4121.5 & 3.1 & 30.4 & 32.1 & 35.6 & \\
\hline $20-056$ & 3.6 & 58.4 & $>7.2$ & 30.1 & 30.5 & $>34.3$ & \\
\hline $22-047$ & 4.0 & 12.7 & $>41.6$ & 30.1 & 30.4 & $>35.1$ & smooth \\
\hline $22-089$ & 2.1 & 9.0 & $>4.2$ & 30.3 & 30.3 & $>34.0$ & smooth \\
\hline $23-002 / 24-002$ & 5.6 & 69.3 & 15.2 & 29.4 & 30.0 & 34.2 & \\
\hline $23-032 / 24-028$ & 2.9 & 39.5 & 46.6 & 30.7 & 30.7 & 35.4 & atypical \\
\hline $23-033 / 24-029$ & 8.7 & 64.9 & 8.6 & 29.2 & 30.0 & 33.9 & \\
\hline $23-045 / 24-038$ & 2.1 & 150.5 & $>26.9$ & 29.6 & 29.6 & $>34.0$ & \\
\hline $23-047 / 24-040$ & 1.6 & 40.3 & 40.9 & 30.9 & 30.7 & 35.3 & atypical \\
\hline $23-047 / 24-040$ & 8.2 & 174.6 & $>17.5$ & 30.9 & 31.6 & $>35.9$ & atypical \\
\hline $23-048$ & 7.1 & 31.3 & $>2.7$ & 28.7 & 29.6 & $>33.0$ & \\
\hline $23-050 / 24-042$ & 13.0 & 637.8 & 47.4 & 30.0 & 30.6 & 35.3 & impulsive \\
\hline $23-063 / 24-055$ & 3.1 & 11.1 & 36.0 & 29.7 & 29.9 & 34.5 & \\
\hline $23-074 / 24-061$ & 1.8 & 8.5 & 6.7 & 30.4 & 30.3 & 34.2 & impulsive \\
\hline $26-067$ & 3.0 & 35.8 & 19.9 & 31.1 & 31.3 & 35.6 & \\
\hline $26-067$ & 2.0 & 23.9 & 9.0 & 31.1 & 31.0 & 35.0 & \\
\hline $26-067$ & 1.7 & 18.3 & 13.0 & 31.1 & 30.9 & 35.0 & \\
\hline $26-072$ & 5.2 & 87.6 & 56.0 & 30.6 & 30.9 & 35.6 & impulsive \\
\hline $28-100$ & 3.2 & 62.2 & 23.8 & 30.2 & 30.4 & 34.8 & impulsive \\
\hline
\end{tabular}

Table 3. XEST X-ray variability statistics for different types of young stars in the broad, soft, and hard bands derived with the MLB technique. For each energy band the number of detected sources, the number of variable sources, and the number fraction of variables are given; see text for details.

\begin{tabular}{|c|c|c|c|c|c|c|c|c|c|c|}
\hline \multirow{3}{*}{$\begin{array}{l}\text { Object } \\
\text { type }\end{array}$} & \multirow{3}{*}{$\begin{array}{r}\text { Type } \\
\text { identifier }\end{array}$} & \multicolumn{3}{|c|}{$\overline{\text { Broad band: } 0.3-7.8 \mathrm{keV}}$} & \multicolumn{3}{|c|}{ Soft band: $0.3-1.0 \mathrm{keV}$} & \multicolumn{3}{|c|}{ Hard band: $1.0-7.8 \mathrm{keV}$} \\
\hline & & \multicolumn{2}{|c|}{ Number } & \multirow{2}{*}{$\begin{array}{l}\text { Fraction } \\
\text { variable }\end{array}$} & \multicolumn{2}{|c|}{ Number } & \multirow{2}{*}{$\begin{array}{c}\text { Fraction } \\
\text { variable }\end{array}$} & \multicolumn{2}{|c|}{ Number } & \multirow{2}{*}{$\begin{array}{c}\text { Fraction } \\
\text { variable }\end{array}$} \\
\hline & & detected & variable & & studied & variable & & studied & variable & \\
\hline Protostar & 1 & $\overline{9}$ & 4 & $44 \%$ & 7 & $\overline{0}$ & $0 \%$ & $\overline{9}$ & 4 & $44 \%$ \\
\hline cTTS & 2 & 52 & 28 & $54 \%$ & 50 & 9 & $18 \%$ & 52 & 27 & $52 \%$ \\
\hline wTTS & 3 & 51 & 26 & $51 \%$ & 51 & 19 & $37 \%$ & 51 & 29 & $57 \%$ \\
\hline Brown dwarf & 4 & 8 & 1 & $13 \%$ & 7 & 0 & $0 \%$ & 7 & 0 & $0 \%$ \\
\hline HAeBe & 5 & 2 & 2 & $100 \%$ & 2 & 2 & $100 \%$ & 2 & 1 & $50 \%$ \\
\hline Unknown & 9 & 4 & 4 & $100 \%$ & 0 & 0 & - & 0 & 0 & - \\
\hline Total & & 126 & 65 & $52 \%$ & 117 & 30 & $26 \%$ & 121 & 61 & $50 \%$ \\
\hline
\end{tabular}

groups: the first group is given by typical or "impulsive" flares with short rise and longer decay. Some flares are termed "atypical" because they show a gradual rise. The group labeled "smooth" in Table 2 is defined by light curves that are either slowly decaying or slowly rising throughout the whole observation. For these sources the characteristic level determined with the MLB procedure is most likely too high. We did not attempt to classify the flares on the fainter stars. In many cases they are described by a single elevated block in the MLB analysis, such that their shape is difficult to determine.

\section{Variability statistics}

Table 3 summarizes the variability statistics for different classes of young stars: protostars, cTTS, wTTS, brown dwarfs (BDs), Herbig Ae/Be (HAeBe) stars, and objects with uncertain classification. The integer numbers for the object type identifiers (Col. 2) have been introduced by Güdel et al. (2007). We examined the variability in the soft and hard bands, analogously to the broad band. Note that in some of the subgroups the sample size (the column labeled "Number studied") is slightly smaller in the restricted energy bands due to insufficient statistics. 
Table 4. XEST X-ray flare statistics for different types of young stars in the $0.3-7.8 \mathrm{keV}$ broad band derived with the MLB technique. For each YSO class, the number of detected sources and the number of flares are given, as is the fraction of flares with respect to the total sample.

\begin{tabular}{lcrrr}
\hline \hline $\begin{array}{l}\text { Object } \\
\text { type }\end{array}$ & $\begin{array}{c}\text { Type } \\
\text { identifier }\end{array}$ & \multicolumn{2}{c}{ Members } & Fraction \\
detected & flare & flare \\
\hline Protostar & 1 & 9 & 2 & $22 \%$ \\
cTTS & 2 & 52 & 16 & $31 \%$ \\
wTTS & 3 & 51 & 11 & $22 \%$ \\
Brown dwarf & 4 & 8 & 0 & $0 \%$ \\
HAeBe & 5 & 2 & 2 & $100 \%$ \\
Unknown & 9 & 4 & 2 & $50 \%$ \\
\hline Total & & 126 & 33 & $26 \%$ \\
\hline
\end{tabular}

Table 5. XEST X-ray variability statistics for different spectral types in the $0.3-7.8 \mathrm{keV}$ broad band; see Tables 3 and 4, and the text in Sect. 4 for details.

\begin{tabular}{lrrrrr}
\hline \hline Spectral & \multicolumn{3}{c}{ Members } & \multicolumn{2}{c}{ Fraction } \\
type & detected & variable & flare & variable & flare \\
\hline BAF & 2 & 2 & 2 & $100 \%$ & $100 \%$ \\
G & 4 & 4 & 3 & $100 \%$ & $75 \%$ \\
K & 35 & 23 & 12 & $66 \%$ & $34 \%$ \\
M & 78 & 32 & 14 & $41 \%$ & $18 \%$ \\
Unknown & 7 & 4 & 2 & $57 \%$ & $29 \%$ \\
\hline Total & 126 & 65 & 33 & $52 \%$ & $26 \%$ \\
\hline
\end{tabular}

In the broad band, variability is detected in about half of the time series. This result does not seem to depend on the object type. In particular, roughly the same fraction of cTTS and wTTS are variable. For the HAeBe stars the number of objects is too small to draw statistically valid conclusions. The absence of variability on 7 of the 8 detected BDs may be explained by their faintness that makes it difficult to discern small-amplitude variations (see Sect. 4.1).

The number and the number fraction of variable sources in the hard band are similar to those in the broad band, but significantly fewer variations are detected in the soft band $(<30 \%$ of the total sample are variable at $0.3-1.0 \mathrm{keV}$ ). This suggests a tight relation between variability and heating processes. On the other hand, in some cases the detection of variability in the soft band may also be impeded by extinction. The visual absorption $A_{\mathrm{V}}$ is on average larger for the sources that show variability in the hard but not in the soft band ("group H"), with respect to the sources that are variable at both soft and hard energies ("group $\mathrm{H}+\mathrm{S}$ "). In particular, $\sim 40 \%$ of the sources from group " $\mathrm{H}$ " have $A_{\mathrm{V}}>4 \mathrm{mag}$, compared to only $\sim 10 \%$ of the sources from group " $\mathrm{H}+\mathrm{S}$ ".

Table 4 summarizes the flare statistics for the different types of young stars. The flare detection process was run only for the broad band. A fraction of $20-30 \%$ of each YSO class with sufficient statistics has shown detectable flares. The flare frequency is marginally larger for cTTS with respect to wTTS, consistent with earlier results based on ROSAT data (Stelzer et al. 2000).

Analogous to the different YSO types, we compare the variability and flare statistics of stars with different spectral types in Table 5. Only the results for the broad band are shown. The fraction of variable stars drops along the spectral type sequence. However, below we show that this is due to an observational bias rather than a physical effect.

\subsection{Observational biases}

Next to residual uncertainties in the background subtraction, the capability to detect variations is affected by Poisson statistics and the inhomogeneous length of the XEST observations. The resulting biases are discussed by investigating the amplitudes of the blocked light curves.

We define the amplitude of the light curves in absolute terms as $A_{\text {abs }}=R_{\max }-R_{\mathrm{ch}}$, where $R_{\max }$ is the highest block count rate. The left diagram in Fig. 3 shows the $A_{\text {abs }}$ of all variable stars with known spectral type as a function of the characteristic rate. Clearly, the absolute amplitude is correlated with $R_{\mathrm{ch}}$. The existence of a lower envelope is due to the statistics that impose a sensitivity limit on the detection of variability. To be recognized as variable, the signal $S_{i}$ in a given block must exceed a certain signal-to-noise level, $\kappa$ :

$S_{i}=N_{i}-N_{\mathrm{ch}, i}>\kappa \sigma\left(N_{\mathrm{ch}, i}\right)=\kappa \sqrt{N_{\mathrm{ch}, i}}$,

where $N_{i}$ is the observed number of counts in block $i, N_{\mathrm{ch}, i}$ is the number of counts in block $i$ that represent the characteristic level, and $\sigma$ is the standard deviation as a measure for the noise. Transformed to count rates, this yields, for the absolute amplitude,

$A_{\mathrm{abs}}=\frac{1}{t_{\max }} \cdot\left(N_{\mathrm{max}}-N_{\mathrm{ch}, \max }\right)>\kappa \sqrt{\frac{1}{t_{\max }}} \cdot \sqrt{R_{\mathrm{ch}}}$.

This sensitivity limit is approximated by the dashed line in Fig. 3. Its location is not uniquely determined because it depends on the duration of the block with the maximum count rate. For reasonable values of $t_{\max } \sim 5 \ldots 20 \mathrm{ks}$, the plotted line corresponds to $\kappa \sim 3.5 \ldots .7$.

On the right hand side of Fig. 3 the relative amplitude, $A_{\text {rel }}=\left(R_{\max }-R_{\mathrm{ch}}\right) / R_{\mathrm{ch}}$ is shown. In this representation the sensitivity threshold has a functional dependence of $1 / \sqrt{R_{\mathrm{ch}}}$. Therefore, events that are small in relative terms are more easily detected on bright stars, while events that are small in absolute terms are more easily detected on faint stars.

An approximate empirical upper envelope to the observed variations is marked in Fig. 3 with a dotted line. In contrast to the lower threshold that is - as explained above - induced by our sensitivity limit, the absence of large amplitudes is probably the result of the intrinsically rare occurrence of strong intensity changes combined with our limited observing time. During the XEST the majority of TMC members did not undergo variations by more than a factor of 8 in count rate.

The different plotting symbols in the right diagram of Fig. 3 distinguish not spectral types but the YSO classes from Table 3. An exceptionally large amplitude is shown by IRAS 04108+2803 B (XEST-20-022), a Class I protostar. This object has shown a spectacular flare near the end of the observation, with a rise in count rate by a factor of $\sim 80$. Its broad band light curve is displayed in Fig. 4. The pre-flare quiescent count rate of IRAS $04108+2803 \mathrm{~B}$ is $\sim 1.9 \times 10^{-3} \mathrm{cps}$ in EPIC/pn, near the detection limit of the observation. According to the spectral analysis carried out by Güdel et al. (2007), the source suffers from strong extinction $\left(\log N_{\mathrm{H}}\left[\mathrm{cm}^{-2}\right] \sim 22.9\right)$. Indeed, the soft emission is completely absorbed, explaining the sharp contrast.

Combining the statistical sensitivity threshold with the empirical maximum of the observed amplitudes, the dynamical range for detectable variations is much larger for bright stars than for faint stars. Therefore, one expects to find more variables among stars with high characteristic emission. To demonstrate the distribution of $R_{\mathrm{ch}}$ in the total sample, at the bottom of Fig. 3 



Fig. 3. Absolute amplitude (left) and relative amplitude (right) of the segmented light curve vs. characteristic count rate for variable stars. On the left the different plotting symbols distinguish stars with different spectral types, on the right, stars of the different YSO classes. The dashed line denotes the sensitivity limit according to Poisson statistics for $\kappa / \sqrt{t_{\max }}=0.05$. The dotted line marks the approximate location of an empirical upper envelope for the amplitudes at $R_{\max }=8 R_{\mathrm{ch}}$. Upward pointing arrows are positioned at the average count rate of non-variable stars of the different spectral types and YSO groups, respectively.

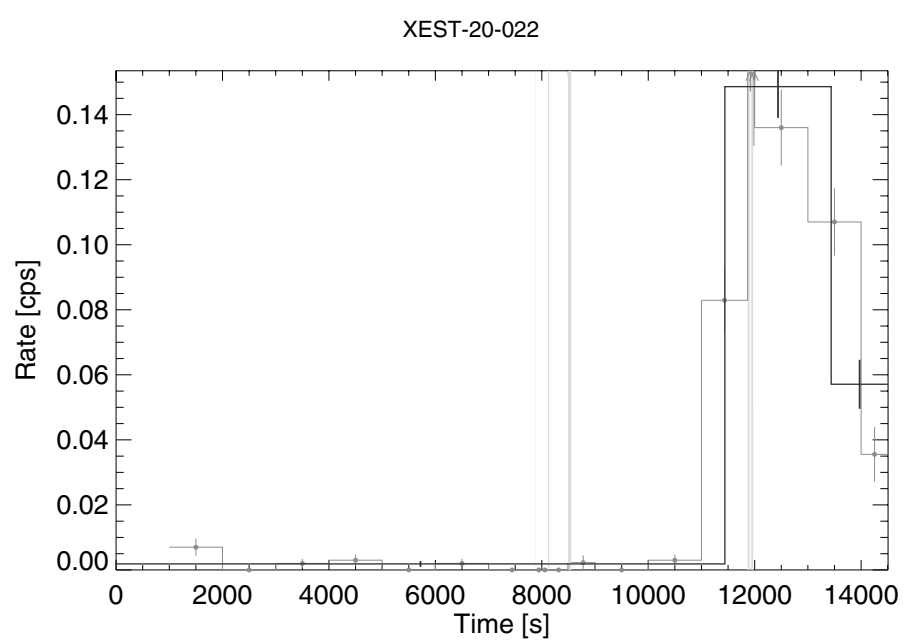

Fig. 4. Background subtracted broad band light curve of the protostar IRAS-04108+2803 B (XEST-20-022) showing a large flare. The data is dominated by the hard band. The soft emission is absorbed due to high column density.

the positions of non-variable stars of the different subgroups are indicated. For $R_{\mathrm{ch}}>0.3 \mathrm{ct} \mathrm{s}^{-1}$ about $50 \%$ of the sample is variable. Similar observations have been discussed in the literature, e.g., Stelzer et al. (2005), Flaccomio et al. (2006) have shown that for a sample in the field of a given X-ray observation all stars above a certain number of counts are variable. Longer observations would probably yield the same result for the TMC sample.

\subsection{Variability of different groups of pre-MS stars}

Taking into account the biases discussed above, we can proceed to a further examination of the variability on different subsamples of TMC members. A comparison of Table 5 with Fig. 3 (left) suggests that the smaller fraction of variables among $\mathrm{M}$ stars when compared to $\mathrm{K}$ stars is a result of their fainter characteristic emission. Indeed, the vast majority of $\mathrm{M}$ stars gather at $R_{\mathrm{ch}}<0.01 \mathrm{ct} \mathrm{s}^{-1}$, where the detection of small variations is impeded by Poisson statistics. The $\mathrm{K}$ stars follow a wider distribution including higher values of $R_{\mathrm{ch}}$, such that the detection of variability is favored. When only sources with $R_{\mathrm{ch}}<0.02 \mathrm{ct} \mathrm{s}^{-1}$ are considered, the fraction of variable sources is $46 \pm 19 \%$ for the $\mathrm{K}$ stars, and the fraction of variables is $50 \pm 12 \%$ for the $\mathrm{M}$ stars, i.e., the variability statistics for the two spectral type classes are indistinguishable.

In an analogous way, the variability statistics of cTTS and wTTS can be compared. It was repeatedly shown in the literature (Neuhäuser et al. 1995; Stelzer \& Neuhäuser 2001) that wTTS are on average X-ray brighter than CTTS. This has also been confirmed for the XEST (Güdel et al. 2007), and therefore one may expect to find a reduced fraction of variable sources among cTTS with respect to wTTS. However, from Table 3 it results that a similar fraction of cTTS and WTTS $(\sim 50 \%)$ is variable. As seen from Fig. 3 (right), both sub-groups have a large fraction of faint objects on which variability is difficult to detect ( $\sim 65 \%$ of all cTTS and $45 \%$ of all wTTS have $R_{\mathrm{ch}}<0.02 \mathrm{ct} \mathrm{s}^{-1}$ ). In the faint subsample with $R_{\mathrm{ch}}<0.02 \mathrm{ct} \mathrm{s}^{-1}$, the fraction of variable sources among the cTTS is $41 \pm 11 \%$, and the fraction of variables among the wTTS is $26 \pm 11 \%$. This is a significant reduction with respect to the sample without restriction in $R_{\mathrm{ch}}$, but the results for cTTS and wTTS are indistinguishable within the statistical uncertainties.

From Fig. 3 it can also be seen that the non-detection of variability on most of the BDs is likely to be related to their 


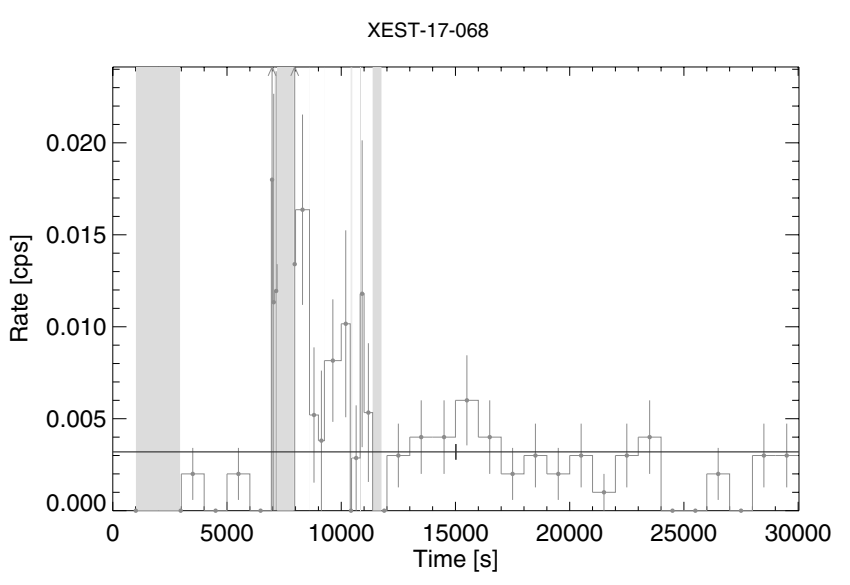

Fig. 5. Background subtracted broad band EPIC/pn light curve of the BD CFHT-BD-Tau 1 (= XEST-17-068). There is some evidence of the possible flare reported by Grosso et al. (2007) at $t \sim 7000-10000 \mathrm{~s}$.

low characteristic count rates: The XEST is sensitive only to large, and presumably rare, flares on the BDs. Grosso et al. (2007) have identified a flare in the binned light curve of CFHTBD-Tau 1 (XEST-17-068). Our MLB algorithm does not recover this event, although there is some evidence of variability in the binned light curve. According to the KS test, the broad band photon time series of XEST-17-068 is variable at the $99 \%$ confidence level. Possibly this flare was missed in the MLB analysis due to high background. Its broad band light curve, displayed in Fig. 5, shows that part of the flare was removed from the data with our high-background filter.

\section{Flare duration and frequency}

The duration of the observed flares ranges from a few ks to $\sim 60 \mathrm{ks}$, with a clustering of events near $\sim 10 \mathrm{ks}$. Obviously, the XEST is biased against the detection of long-duration events, due to the short exposure times (typically $\sim 30 \mathrm{ks}$ ). Recall that the distribution of flare durations derived from the $13 \mathrm{~d}$-long COUP exposure (Wolk et al. 2005) ranges from $1 \mathrm{~h}$ to $3 \mathrm{~d}$ with a peak near $18 \mathrm{~h}$. Consequently - leaving apart subtle differences in the definition of a flare in the work by Wolk et al. (2005) and the one presented here, and ignoring that the two studies concern stars of different mass ranges that may influence the flare characteristics - it is no surprise that nearly half of the flares we observed in the XEST are only partly within the observing time. As mentioned above we can give only lower limits on the duration of these events.

The frequency of flares for the total XEST sample of TMC members computed from the total observing time $T_{\text {obs }}$ and the number of observed flares is 1 flare per star in $200 \mathrm{ks}$. In computing this number we have assumed that we are sensitive to the detection of flares throughout the whole observing time. This should be a reasonable approximation because observing gaps due to high background are mostly shorter than the typical duration of the flares. A serious limitation, however, comes from the fact that we are comparing stars whose characteristic count rates differ by as much as a factor of 1000 . As a consequence the events detected in our observations form an incomplete subsample of the total flare population. In Sect. 6 we argue that the flare detection is complete for events with $E_{\mathrm{F}}>10^{35} \mathrm{erg} / \mathrm{s}$. If only those events are considered, the flare frequency of the TMC members reduces to 1 flare in $770 \mathrm{ks}$. Our flare detection is also biased by the limited length of the XEST observations, typically $\sim 30 \mathrm{ks}$. Indeed, during the long $(115 \mathrm{ks})$ merged observation XEST-23/XEST-24, 8 flares were identified on the 18 stars detected in both exposures XEST-23 and XEST-24. Counting only the events with energy above $10^{35} \mathrm{erg}$, the XEST-23/XEST24 field has a flare frequency of 1 in $520 \mathrm{ks}$.

These numbers can be compared to the flare frequency observed during the COUP for the young solar-analogs in the ONC. There are 30 flares that emit more than $10^{35} \mathrm{erg} / \mathrm{s}$ (Table 6 of Wolk et al. 2005), and - following the discussion in the COUP article - we estimate that their occurrence rate is $\sim 1 / 900 \mathrm{ks}$, similar to our result for a sample of wider mass range in the TMC.

The fraction of the total observing time during which a given group of stars was found in the flare state is defined by

$$
F_{\mathrm{F}}=\frac{\sum_{i} \tau_{i}}{T_{\mathrm{obs}}}
$$

where the sum goes over the durations of all flares. For the total TMC sample we find $F_{\mathrm{F}} \sim 8 \%$. For the subgroups of cTTS and wTTS the fractional time in the flare state is found to be indistinguishable in both cases at $\sim 8-9 \%$. Stelzer et al. (2000) have found $\sim 1 \%$ for the flare rate of TMC stars, evaluating pointed ROSAT observations. This discrepancy may indicate that ROSAT missed a substantial number of flares due to gaps in the observing sequence resulting from Earth occultations, or due to uncertainties in the flare durations related to these gaps, or due to the smaller effective area or missing sensitivity for more energetic photons.

\section{Flares and the coronal heating process}

To study the effect of the flare population on coronal heating, we now construct the distribution of flare occurrence rate in total released energy. For the Sun, it was shown that this distribution follows a power law (e.g., Hudson 1991)

$$
\frac{\mathrm{d} N}{\mathrm{~d} E} \sim E^{-\alpha}
$$

The radiated energy released in flares in the observed energy band is obtained by integrating this differential distribution. Generally, observational studies are affected by a sensitivity limit that impedes the detection of very small flares. It is common practice to extrapolate the power law to energies below the detection threshold to get a hold of the energy in small unresolved events. Such "nano-flares" are assumed to exist because they can be considered as the heating agent that gives rise to the quiescent corona of the Sun and other magnetically active stars. If the power law index $\alpha>2$, very small flares can in principle contribute an unlimited amount of energy, because the integral of Eq. (6) diverges for $E_{\min } \rightarrow 0$. Clearly, in such cases there must either be a lower cut-off to the flare energies, or the power law must turn over to $\alpha<2$ at low energies.

Although we are limited to measurements exceeding $\log E_{\mathrm{F}}$ [erg] $\approx 33.5$ in our survey, we note that a power law distribution has been followed down to luminosities of $\log E_{\mathrm{F}}[\mathrm{erg}]=$ 30.5 for a few examples of somewhat more evolved, magnetically active low-mass stars, and to levels as low as $\log E_{\mathrm{F}}$ [erg] = 25 in the solar corona. For the Sun the situation has remained inconclusive, with $\alpha$ between $1.6-2.6$ over a wide range of energies (Crosby et al. 1993; Krucker \& Benz 1998; Aschwanden et al. 2000). For stars, the hard X-ray range, mostly used in solar flare observations, is inaccessible. In recent statistical studies of EUV flare energy distributions on magnetically active stars, 
Audard et al. $(1999,2000)$ find power law indices close to the critical value $(\alpha \sim 2)$. Further observations of stellar flare energy distributions are summarized by Kashyap et al. (2002) and Güdel et al. (2003), who point out that the slope may depend on the spectral range of the instrument. A comparison of the distributions observed in the EUV, in soft, and in hard X-rays showed that these different temperature regimes correspond to different energy ranges from "nano"- to "milli-flares", and that they represent different density, pressure, and emission measure regimes (Aschwanden et al. 2000). Therefore, it is unclear if the extrapolation from the large observable flares toward the lowest energies is valid.

The analysis of such distributions is subject to several caveats. One of them is that small flares overlapping larger ones are masked and missed in the flare detection process. Audard et al. (2000) have mediated this problem by applying a correction factor to the flare rate at each energy. This correction takes into account the fact that the "effective" time available for the identification of small flares is given by the total observing time $\left(T_{\mathrm{obs}}\right)$ reduced by the sum of the durations of larger flares $\left(\tau_{>\mathrm{E}}\right)$, such that the flare rate at energy $E$ must be multiplied by a factor

$f_{\mathrm{E}}=T_{\mathrm{obs}} /\left(T_{\mathrm{obs}}-\tau_{>\mathrm{E}}\right)$.

\subsection{Flare energy distributions: TMC and ONC}

Systematic studies of the energy release during flares on preMS stars have been carried out for only two star-forming regions so far: the TMC (this study) and the solar-analogs of the ONC (Wolk et al. 2005). In Fig. 6 the cumulative number distributions $\left[N\left(>E_{\mathrm{F}}\right)\right]$ of flares observed in both samples are shown in double logarithmic form.

We derived the distribution of the ONC from the mean flare energies given in Table 6 of Wolk et al. (2005). In contrast to our XEST study of the TMC, these energies were computed from the spectrum during the flare state. In some cases the values for $E_{\mathrm{F}}$ are dubious as a result of poor spectral fits, and we excluded events flagged with "c", "e", or "f" in Table 6 of Wolk et al. (2005) from the analysis of the energy distribution.

For both distributions, we performed the correction for overlapping flares described above by multiplying each value of $N(>E)$ with its appropriate factor $f_{\mathrm{E}}$. For the ONC data we assumed a total observing time of $850 \mathrm{ks} \cdot 22$, where 22 is the number of stars in the sample after removing the 5 stars with poorly defined flares mentioned above. The duration of each flare is taken from Table 6 of Wolk et al. (2005). The effect of the correction factor $f_{\mathrm{E}}$ is to steepen the slope, but in practice its influence is negligible because the flare frequency is low, such that $\tau_{>\mathrm{E}}$ is only a minor fraction of $T_{\text {obs }}$.

The faintest flare detected in the ONC sample has an energy of $10^{34.5} \mathrm{erg} / \mathrm{s}$, while in the TMC we have access to events that are one order of magnitude lower. This difference in sensitivity corresponds roughly to the difference in X-ray flux for a source of given luminosity that arises from the distance ratio $\left(d_{\mathrm{ONC}} / d_{\mathrm{TMC}}\right)^{2}(140 \mathrm{pc}$ for TMC vs. $450 \mathrm{pc}$ for the ONC).

\subsection{Determination of the power law slope}

As discussed above, the distribution of flare energies is expected to follow a power law. However, the observed distributions flatten towards lower energies. This saturation is probably an observational bias, indicating that smaller events are missed by the flare detection process. On the other hand, the steep high-energy portion represents large flares that are readily detectable, such

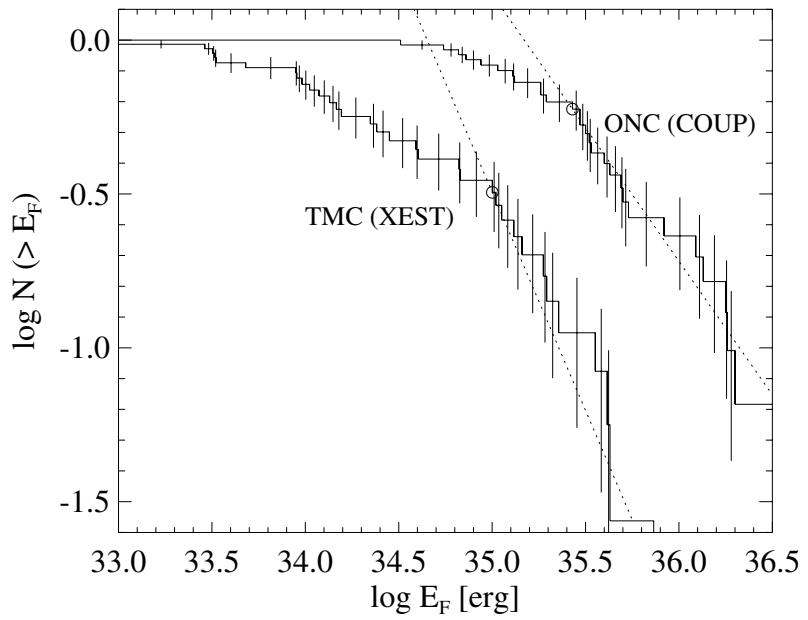

Fig. 6. Cumulative distribution of flare energies for the pre-MS sample in the TMC observed during the XEST (Table 2) and for the solaranalogs of the ONC observed during the COUP (Table 6 of Wolk et al. 2005). The dotted lines represent the ML estimates for the slope of the distribution $\log N=\beta \cdot \log E+C$ in the range of energies above the cutoff value $E_{\text {cut }}$ indicated by the open circle.

that above a certain threshold energy $E_{\text {cut }}$ the flare identification is likely complete.

We have determined both the slope $\beta$ of the function $\log N\left(>E_{\mathrm{F}}\right)=-\beta \cdot \log E_{\mathrm{F}}+C$ and the cutoff energy $E_{\text {cut }}$ with a maximum likelihood method. According to Crawford et al. (1970) the ML estimate for the power law slope is

$\frac{1}{\beta}=\frac{1}{N} \sum_{i} \ln \left(\frac{E_{i}}{E_{\text {cut }}}\right)$,

where $N$ is the total number of flares above $E_{\text {cut }}$, and $E_{i}$ are their energies. To test if Eq. (8) is a good representation of the data we have performed the transformation

$y_{i}=\left(1-\left(\frac{E_{i}}{E_{\text {cut }}}\right)^{-\beta}\right) /\left(1-\left(\frac{E_{\text {max }}}{E_{\text {cut }}}\right)^{-\beta}\right)$

and tested this distribution against departure from a uniform distribution using the KS test (Crawford et al. 1970).

We have evaluated Eqs. (8) and (9) for a range of cutoff energies. The result is shown in Fig. 7. It presents in the same graph, but with different scales, the run of $\beta$ (filled circles) and the probability $P_{\mathrm{KS}}$ from the KS test (open squares). High values of $P_{\mathrm{KS}}$ indicate that the distribution of $y_{i}$ is compatible with the null hypothesis of being uniform in the interval $[0,1]$. Therefore, the power law approximation of the observed flare energy distribution is justified for cutoff energies in the peak of $P_{\mathrm{KS}}\left(\mathrm{E}_{\mathrm{cut}}\right)$. From Fig. 6 the power law index $\beta$ is expected to increase with increasing $E_{\text {cut }}$. For cutoff energies where the flare energy distribution is well represented by a power law (i.e., for high values of $P_{\mathrm{KS}}$ ), the flare detection process is probably complete. Therefore, further increasing $E_{\text {cut }}$ should not change the slope, and $\beta\left(\mathrm{E}_{\text {cut }}\right)$ reaches a plateau. Obviously, at the largest cutoff energies the result is compromised by low-number statistics. The number of flares with $E_{\mathrm{F}}>E_{\text {cut }}$ are given on the top of both panels in Fig. 7.

\subsection{Results and caveats}

The best match for the power law index determined from Fig. 7 is $\beta_{\mathrm{TMC}}=1.4 \pm 0.5$ for the TMC and $\beta_{\mathrm{ONC}}=0.9 \pm 0.2$ for the 

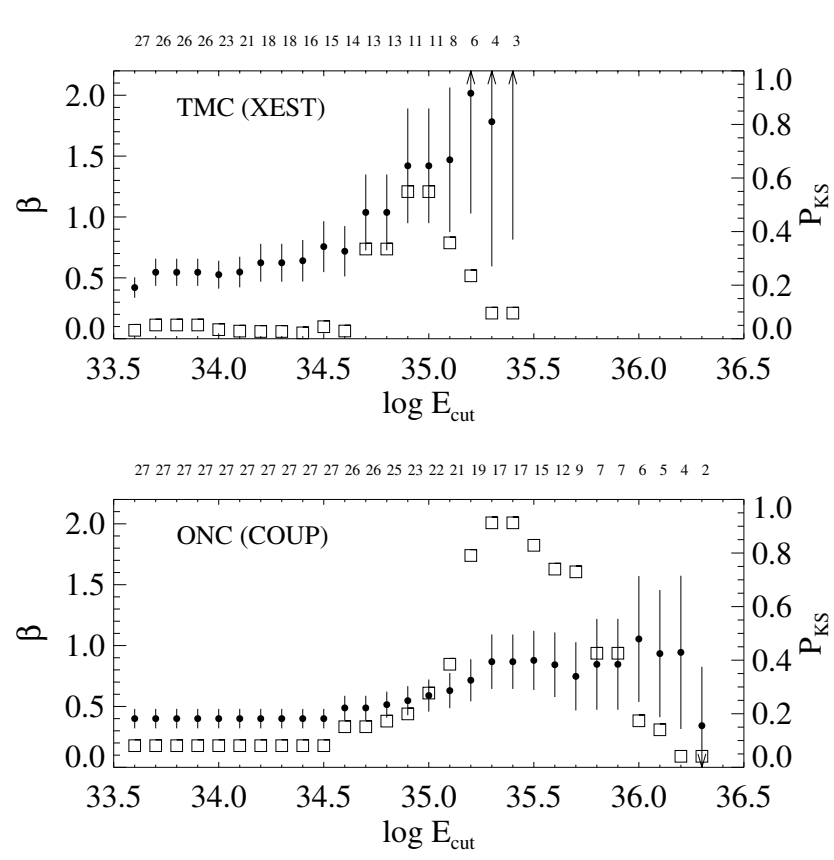

Fig. 7. Result from the ML estimate of the power law index for the flare energy distribution of the XEST sample in the TMC and the COUP sample in the ONC as a function of minimum flare energy included $\left(E_{\text {cut }}\right)$ : filled circles - slope $\beta$ of the double logarithmic cumulative distribution of flare energies $(\log N=-\beta \cdot \log E+C)$; open squares - probability of KS test that the above power law is a good approximation of the observed flare energy distribution.

ONC. These slopes are plotted in Fig. 6, and the corresponding $E_{\text {cut }}$ is marked with an open circle. It suggests that the flare detection is complete for events of $\log E_{\mathrm{F}}$ [erg] $>34.9$ in the case of the TMC, and $\log E_{\mathrm{F}}$ [erg] $>35.3$ for the ONC. The cumulative distribution of Fig. 6 has the derivative $\mathrm{d} N / \mathrm{d} E_{\mathrm{F}} \sim E_{\mathrm{F}}^{-\alpha}$, where $\alpha=1+\beta$. Therefore, from our observations we find $\alpha_{\mathrm{TMC}} \sim 2.4$ and $\alpha_{\mathrm{ONC}} \sim 1.9$. Both values are consistent with each other and with $\alpha>2$, considering the substantial uncertainties.

Besides the statistical errors, there are various uncertainties and differences in the approach between the ONC and the TMC sample, e.g., the definition of the flares and the evaluation of the flare energies. In our analysis we have ignored that the observed energies for some flares in the XEST are lower limits. No information on a possible incomplete coverage of the ONC flares is given by Wolk et al. (2005). However, as judging from their Fig. 3, some of the events should be flagged lower limits because data gaps during the flare have caused a loss of photons for the spectral analysis. We have verified in simulations that the power law index $\beta$ is not affected by ignoring the censoring of some energies. If a certain number of values at random drawn from a power law distribution are decreased by a random fraction of their original value, this results in a leftward shift of the cumulative number distribution, but the slope remains unmodified. This means that we implicitly assume a random distribution of the lower limits across the range of observed flare energies. While in practice this assumption remains unproven, this is unlikely to be of major importance to our results.

We also note that we did not perform a thermal modeling of individual flares to estimate their radiated energies in detail, given that the spectra were too faint in many cases to obtain reliable temperature, emission measure, and absorption parameters.

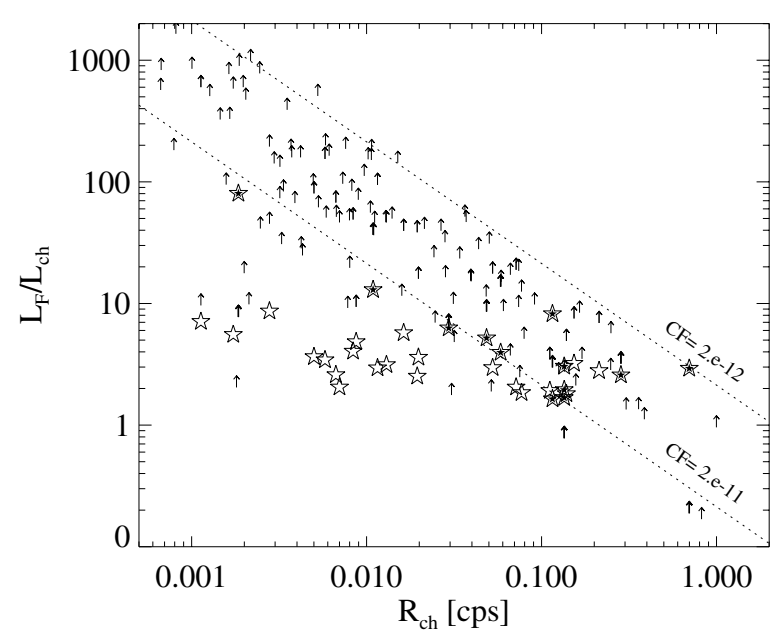

Fig. 8. Flare sensitivity limit. For each star, is shown the amplitude above which all flares with an assumed emitted energy of $10^{35} \mathrm{erg}$ and a duration of $10 \mathrm{ks}$ are detectable (upward pointing arrows). The conversion from count rate to luminosity depends on the spectral shape and is expressed by the dotted lines that indicate two different count-to-energy conversion factors. Asterisks denote the amplitudes of observed flares; filled asterisks are those flares with $E>10^{35} \mathrm{erg}$, and the flare sensitivity limit of the corresponding star is shown as a thicker arrow.

It was implicitly assumed that the flare parameters are similar to the coronal parameters determined for the overall emission. Given the relatively moderate dynamic range of flare amplitudes and also their moderate amplitudes compared with the overall emission level in the respective light curves, we believe that this approximation is appropriate.

\subsection{Completeness limit for flare detection}

We estimate the minimum signal for the detection of a flare with edge energy $E_{\mathrm{F}, \text { edge }}=10^{35} \mathrm{erg}$ and a typical duration of $10 \mathrm{ks}$. For such events the flare luminosity is $\log L_{\mathrm{F} \text {,edge }}[\mathrm{erg} / \mathrm{s}]=31$. We compute the value of $L_{\mathrm{F} \text {,edge }} / L_{\mathrm{ch}}$ that corresponds to this threshold from the characteristic count rate of each star. Again, we use PIMMS with spectral parameters from Table 6 of Güdel et al. (2007) to determine an individual count-to-flux conversion factor for each source, which allows us to determine $L_{\mathrm{ch}}$.

The resulting amplitude $L_{\mathrm{F} \text {,edge }} / L_{\mathrm{ch}}$ is visualized in Fig. 8 . The choice of upward pointing arrows as a plotting symbol is motivated by the indications discussed in Sect. 6.2 that all flares with energy above the cutoff $E_{\mathrm{F}, \text { edge }}$ are detected, such that $L_{\mathrm{F}, \text { edge }} / L_{\mathrm{ch}}$ represents a completeness threshold. As can be seen, for a given $R_{\mathrm{ch}}$ the differences in the spectral shape result in a spread of more than one order of magnitude in the luminosity amplitude. This is illustrated by the dotted lines that represent two different conversion factors. For comparison, in Fig. 8 the amplitudes of all observed flares are also shown (asterisks). Generally, the "completeness limit" corresponds to much higher amplitudes than observed, leaving space for a large population of undetected events. Filled asterisks denote those flares with $E_{\mathrm{F}}>10^{35} \mathrm{erg}$. As expected most of these are well above the derived completeness limit for their host star (marked with thicker arrows). In some cases they are below, and this can be explained by their duration being much longer than our assumption of $10 \mathrm{ks}$. 




Fig. 9. Flare energy and flare duration for cTTS and wTTS. Lower limits for flares that are unconstrained because partially outside the observing window are indicated with arrows.

\section{Variability of cTTS and wTTS}

A comparison of the variability characteristics of cTTS and wTTS is of interest because of the possibly different emission mechanisms: next to the canonical interpretation of magnetic structures with footpoints anchored on the star, cTTS may produce X-ray emission related to the accretion process and/or in magnetic structures connecting the star with the circumstellar disk. In wTTS the absence of a disk and accreting material imply that the most plausible explanation of the short-term X-ray variability is magnetic flaring.

Observationally it is not straightforward to distinguish between variability originating from these different causes, unless there is a sufficient signal for detailed modelling of the spectral evolution that gives access to the strength of the magnetic field and the length scale of magnetic structures (see, e.g., Favata et al. 2005). A few of the brightest XEST sources are examined in this respect by Franciosini et al. (2007). Here we compare the variability of cTTS and wTTS using a statistical approach.

In Sect. 4 we have established that a similar fraction of cTTS and wTTS are variable in the broad band. In the soft band, variability is more frequent on wTTS than on cTTS: $18 \pm 6 \%$ variables among cTTS vs. $37 \pm 9 \%$ variable wTTS. A possible cause for this difference is the on-average larger extinction of the former ones that removes soft photons from the spectrum. About $30 \%$ of all cTTS have $A_{\mathrm{V}}<1 \mathrm{mag}$, compared to more than two-thirds of the wTTS sample. This trend is even more pronounced among the stars that are constant in the soft band, and supports the suspicion that soft absorption puts stronger limits on the detection of variability in cTTS than in wTTS. On the other hand, if a significant amount of X-rays from cTTS were produced from accretion, then at least those cTTS that are not absorbed should show more - not less - soft variability than wTTS because the temperatures in a T Tauri accretion shock (of a few MK at most) can produce only soft photons $(<1 \mathrm{keV})$. This is not observed. An alternative explanation would be that accretion is steady enough not to produce X-ray variability, or that variations in the density of the accreting material are smoothed out during the X-ray generation process (Lamzin 1999).

The XEST observations show further that flares (characterized by large amplitude and fast rise) are identified marginally more frequently on cTTS than on wTTS $(31 \pm 7 \%$ vs. $22 \pm 7 \%)$.
A similar conclusion, but likewise not on a strong statistical basis, was obtained by Stelzer et al. (2000) based on ROSAT observations. In Fig. 9 the flare durations and energies of cTTS and wTTS measured during the XEST are shown. There is not a clear distinction between the two groups, pointing at a similar - most likely coronal - origin for the X-rays from both classes of stars.

\section{Conclusions}

We have presented a systematic analysis of X-ray variability on pre-MS stars in the TMC based on the XEST. This project includes a significant fraction of the known TMC members, and presents the deepest X-ray survey so far in the Taurus starforming region. We have used an automated procedure for detecting variability and recognizing flares. Flares are defined with criteria that take into account the amplitude and the derivative of the segmented light curves. Variability is found in roughly half of the sample in the broad band from $0.3-7.8 \mathrm{keV}$. Our analysis in different energy bands has shown that it is important to include hard energies: Variability is detected more frequently in the hard band $(50 \%$ of sources are variable at $>1 \mathrm{keV})$ than in the soft band $(26 \%$ of sources are variable at $<1 \mathrm{keV})$ where, in some sources, strong extinction lowers the signal to the point that it becomes difficult to reveal variations. Next to this bias there is probably a physical reason behind the fact that variability is stronger at harder energies, related to the notion that variability goes along with additional heating.

The detection of variability is biased because the Poisson statistics impose different sensitivity thresholds for sources with different count rates, and the sample has a large range of count rates (four orders of magnitude). We have estimated the influence of this bias on the variability statistics and evaluated the variability statistics for different classes of pre-MS stars (protostars, cTTS, wTTS, brown dwarfs) and for different spectral type ranges that roughly correspond to mass for cool pre-MS stars.

Variability is found slightly more frequently on cTTS than on wTTS. The difference is only marginal, but when combined with similar indications from various star-forming regions discussed in the literature (e.g., Flaccomio et al. 2006), this finding may deserve further attention. On the other hand, the flare energies and durations of cTTS and wTTS are similar, and there is no evidence of extra soft variability from accretion in cTTS. All in all, the XEST data does not yield support for models that predict X-ray emission triggered by reconnection related to stardisk magnetospheric interaction and accretion (e.g., Shu et al. 1994; Goodson \& Winglee 1999), although such scenarios may apply in individual cases; see also discussion by Franciosini et al. (2007).

There is no dependence of the fraction of variable sources on spectral type (comparing K and M stars). Calvet et al. (2004) summarized a correlation between mass accretion rate, $\dot{M}$, and stellar mass, $M$, that is found to be $\dot{M} \propto M^{1.95}$. If X-ray production were related to the accretion activity (e.g., through accretion shock heating or through influence on the coronal magnetic structure), then stronger accretion would be expected to produce stronger (absolute) fluctuations, and therefore X-ray variability should be different not only for accreting and non-accreting stars, but also for lower- and higher-mass cTTS in the sample. Since M stars and K stars show no distinction with regard to absolute variability, we find no evidence that the accretion process significantly influences X-ray variability. Preibisch et al. (2005) arrived at similar conclusions for the ONC sample based on a comparison of the thermal properties of cTTS and wTTS. 
We have evaluated the flare rates for the events on TMC members identified in this work, and compared them to equivalent data for the ONC sample obtained by Chandra within the COUP. In both cases the sample represents pre-MS stars, but the ONC study concerns a narrow mass range $\left(0.9 M_{\odot}<M<\right.$ $1.2 M_{\odot}$ ), while in this work we have included all TMC members detected during the XEST. The frequency of large flares $\left(E_{\mathrm{F}}>10^{35} \mathrm{erg}\right)$ in the TMC is 1 event per star in $770 \mathrm{ks}$, roughly comparable to the estimate for the young solar analogs in the ONC.

We have found twofold observational support for coronal heating by flares in our stellar sample. First, we have noted that the flare amplitude is correlated with the characteristic emission level of the flaring star. In particular, we found an upper envelope to the flare amplitudes as a function of $R_{\mathrm{ch}}$. While the envelope is unlikely to be strict because longer observations could detect some of the very rare but very large flares on any star, the trend can naturally be explained if flares are related to the coronal heating process (Audard et al. 2000): One possibility is that both the flare rate and the overall heating are the results of a similar process, probably magnetic reconnection, and therefore both the flare rate for a given flare amplitude and the total X-ray luminosity are expected to scale with the coronal volume available for these processes. Alternatively, the characteristic emission could be the direct result of the heating process of a large number of unresolved flares occurring continuously on the star. Both assumptions relate the physical processes relevant to flares to the overall stellar radiative X-ray losses. In both cases, one expects (for a given observing time) that the maximum of the absolute amplitude be proportional to $R_{\mathrm{ch}}$, and this is indeed what we find (Fig. 3, left).

Secondly, we have analyzed the cumulative number distribution of flare energies, making use of our new results for the TMC and of the flare energies tabulated by Wolk et al. (2005) for the ONC. Our ML estimates show that the distributions of both samples can be represented by a power law, with an index of $\alpha=2.4 \pm 0.5$ for the differential distribution of the TMC, and $\alpha=1.9 \pm 0.2$ for the ONC. Wolk et al. (2005) had provided a value of $\alpha \sim 1.7$ for the same ONC data, using a linear leastsquares fit to the double logarithmic representation of the cumulative number distribution. However, that approach does not take into account that the individual bins are not independent. Therefore, our method is more rigorous. The power law slope for the number distribution of flare energies in the TMC and in the ONC, although not well constrained, is in agreement with the range found in previous studies of solar and stellar flares (see references in Sect. 6), indicating that the energy release in cool stars is a fairly universal process. The observed values for $\alpha$ are near and probably larger than the critical limit $\left(\alpha_{\text {crit }}=2\right)$. Therefore, although the uncertainties preclude definite conclusions, micro-flare heating may well play an important role in heating these pre-MS coronae.

In summary, the entire sample supports an important role of flare-like events in the generation of the X-ray light curves, in a similar manner as observed before in non-accreting magnetically active stars. In particular, we conclude that cTTS and wTTS reveal similar variability characteristics. These results point at magnetic energy release playing the dominant role in the generation of X-rays, while there is little evidence of the influence of accretion disks (via long-scale magnetic fields) or accretion itself (via accretion-shock heating). While neither of the latter two can be excluded from contributing to the observed X-rays, we claim that neither is responsible for strong X-ray losses, but that compact magnetic field annihilation dominates the $\mathrm{X}$-ray production.

Acknowledgements. We warmly acknowledge financial support by the International Space Science Institute (ISSI) in Bern to the XMM-Newton XEST team. B.S., E.F., G.M., L.S., and I.P. acknowledge financial contributions from contract ASI-INAF I/023/05/0. X-ray astronomy research at PSI has been supported by the Swiss National Science Foundation (grants 20-66875.01 and 20109255/1). M.A. acknowledges support by NASA grants NNG05GF92G. B.S. thanks J. Bouvier for stimulating comments on the manuscript. This research is based on observations obtained with $X M M-N e w t o n$, an ESA science mission with instruments and contributions directly funded by ESA member states and the USA (NASA).

\section{References}

Antonucci, E., Gabriel, A. H., \& Dennis, B. R. 1984, ApJ, 287, 917

Aschwanden, M. J., Tarbell, T. D., Nightingale, R. W., et al. 2000, ApJ, 535, 1047

Audard, M., Güdel, M., \& Guinan, E. F. 1999, ApJ, 513, L53

Audard, M., Güdel, M., Drake, J. J., \& Kashyap, V. L. 2000, ApJ, 541, 396

Calvet, N., Muzerolle, J., Briceño, C., et al. 2004, AJ, 128, 1294

Crawford, D. F., Jauncey, D. L., \& Murdoch, H. S. 1970, ApJ, 162, 405

Crosby, N. B., Aschwanden, M. J., \& Dennis, B. R. 1993, Sol. Phys., 143, 275

Favata, F. \& Micela, G. 2003, Space Sci. Rev., 108, 577

Favata, F., Micela, G., Baliunas, S. L., et al. 2004, A\&A, 418, L13

Favata, F., Flaccomio, E., Reale, F., et al. 2005, ApJS, 160, 469

Feigelson, E. D., \& Montmerle, T. 1999, ARA\&A, 37, 363

Flaccomio, E., Micela, G., Sciortino, S., et al. 2005, ApJS, 160, 450

Flaccomio, E., Micela, G., \& Sciortino, S. 2006, A\&A, 455, 903

Franciosini, E., Pillitteri, I., Stelzer, B., et al. 2007, A\&A, 468, 485

Goodson, A. P. \& Winglee, R. M. 1999, ApJ, 524, 159

Grosso, N., Briggs, K., Güdel, M., et al. 2007, A\&A, 468, 391

Güdel, M. 2004, A\&AR, 12, 71

Güdel, M., Audard, M., Skinner, S. L., \& Horvath, M. I. 2002, ApJ, 580, L73

Güdel, M., Audard, M., Kashyap, V. L., Drake, J. J., \& Guinan, E. F. 2003, ApJ, 582,423

Güdel M., Briggs, K., Arzner, K., et al. 2007, A\&A, 468, 353

Hudson, H. S. 1991, Sol. Phys., 133, 357

Kashyap, V. L., Drake, J. J., Güdel, M., \& Audard, M. 2002, ApJ, 580, 1118

Krucker, S., \& Benz, A. O. 1998, ApJ, 501, L213

Lamzin, S. A. 1999, Astron. Lett., 25, 430

Marino, A., Micela, G., Peres, G., \& Sciortino, S. 2003, A\&A, 407, L63

Neuhäuser, R., Sterzik, M. F., Schmitt, J. H. M. M., Wichmann, R., \& Krautter, J. 1995, A\&A, 297, 391

Preibisch, T., Kim, Y.-C., Favata, F., et al. 2005, ApJS, 160, 401

Raymond, J. C., \& Smith, B. W. 1977, ApJS, 35, 419

Scargle, J. D. 1998, ApJ, 504, 405

Shu, F., Najita, J., Ostriker, E., et al. 1994, ApJ, 429, 781

Stelzer, B. \& Neuhäuser, R. 2001, A\&A, 377, 538

Stelzer, B., Neuhäuser, R., \& Hambaryan, V. 2000, A\&A, 356, 949

Stelzer, B., Flaccomio, E., Montmerle, T., et al. 2005, ApJS, 160, 557

Wolk, S. J., Harnden, Jr., F. R., Flaccomio, E., et al. 2005, ApJS, 160, 423 


\section{Online Material}


B. Stelzer et al.: X-ray variability on pre-MS stars in the TMC, Online Material p 3

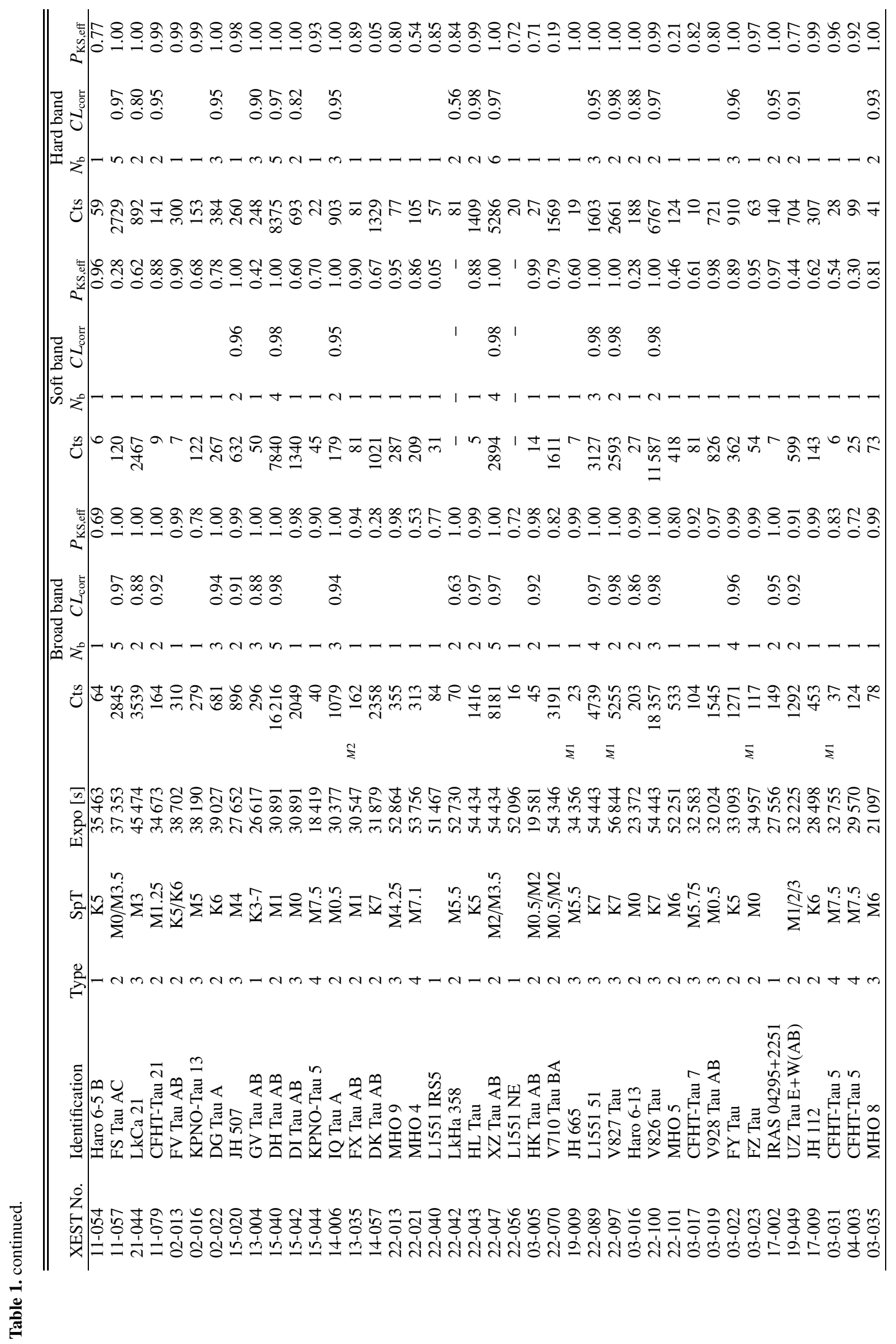




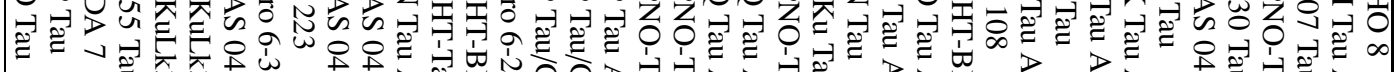

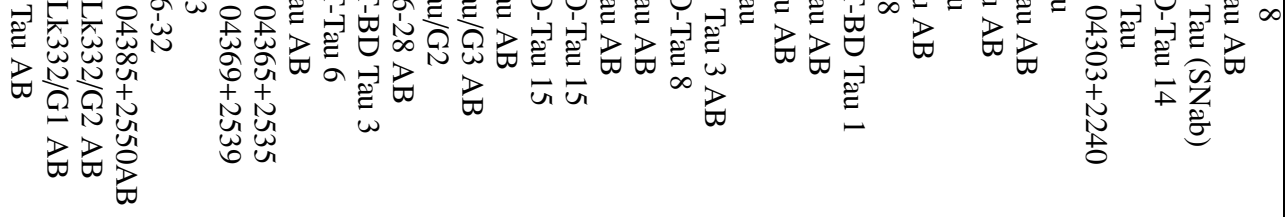
NNNNWWN

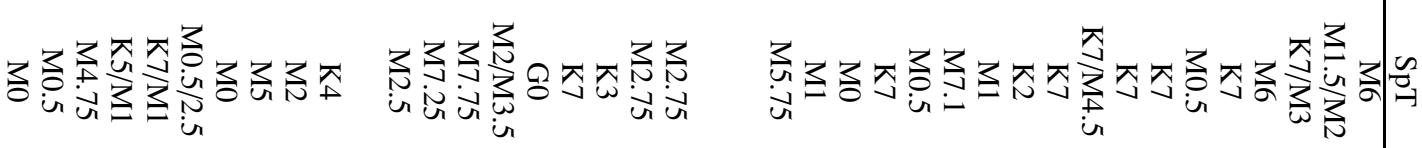
N

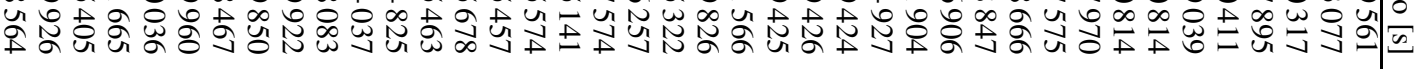
$\Xi$ 3 $\Xi$

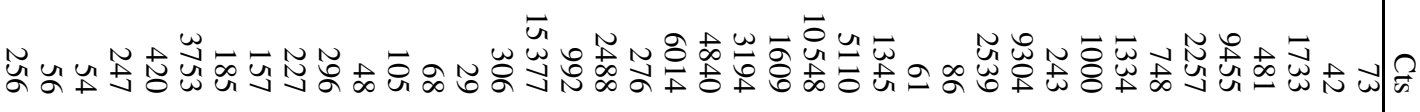

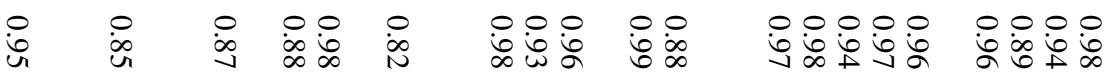

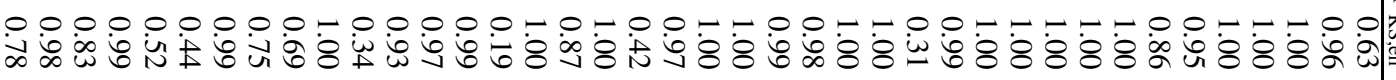

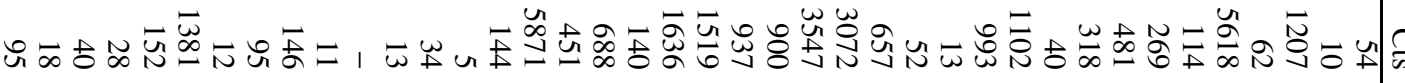

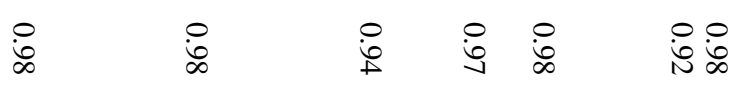



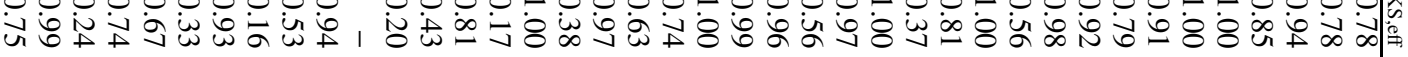

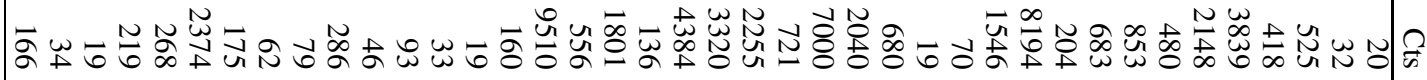

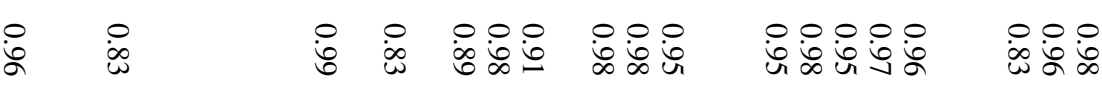

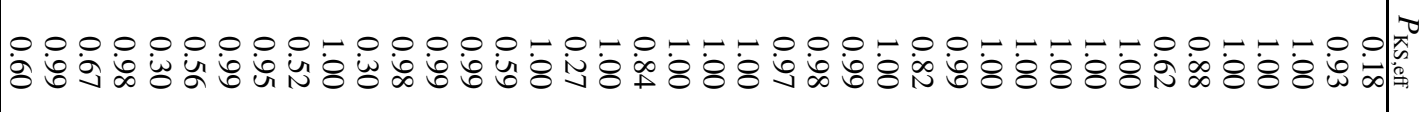




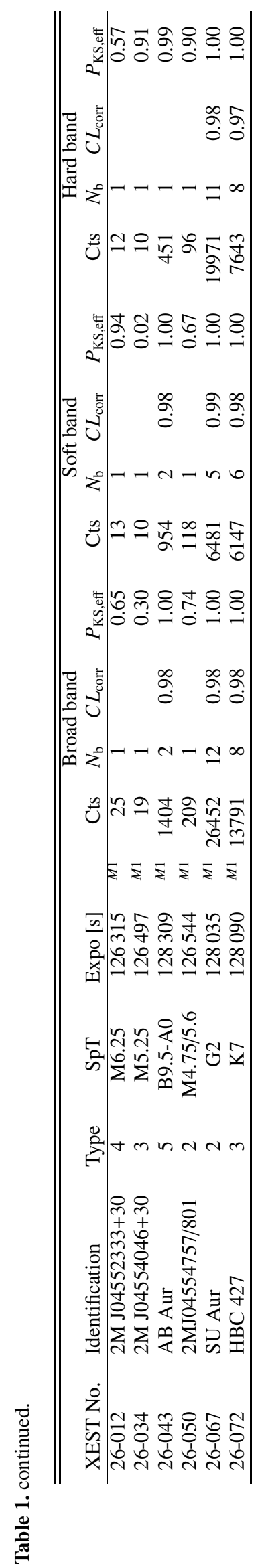

